\title{
Ultrasonido Doppler con contraste para el diagnóstico de cortocircuito de derecha a izquierda: revisión sistemática y metaanálisis
}

Contrast Doppler ultrasound for diagnosis of right to left shunt: systematic review and metanalysis

Ultrassom Doppler de contraste para diagnóstico de curto-circuito da direita para a esquerda: revisão sistemática e metanálise

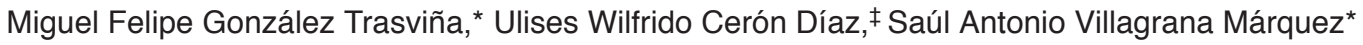

\section{RESUMEN}

Introducción: La comunicación de derecha a izquierda (CDI) se produce principalmente con lesiones intracardiacas, como el foramen oval permeable (FOP) y, en menor medida, a través de malformaciones arteriovenosas pulmonares (MAVP). Desde el informe inicial de Gramiak y colaboradores, la ecocardiografía con contraste ha sido el método de elección para identificar $\mathrm{CDI}$, siendo el ecocardiograma transesofágico con contraste (ETE-c) actualmente el método diagnóstico ideal para $\mathrm{CDI}$. Material y métodos: Se realizó una revisión sistemática con el objetivo de conocer el rendimiento diagnóstico del ultrasonido Doppler contrastado (UD-c) en sus diferentes variantes (transcraneal, vertebral y carotídeo) en comparación con el ETE-c para el diagnóstico de CDI; la revisión se realizó en las bases de datos EBSCO, Elsevier, KoreaMed, Lilacs, PubMed/Medline, OVID/Cochrane Library, Scielo, SCOPUS, Web of Science y EMBASE.

Resultados: Se obtuvieron 33 publicaciones que cumplían con los criterios de inclusión, aportando 36 estudios con 3,099 participantes (edad promedio de 47.1 años; $53 \%$ masculinos). De éstos, sólo se incluía un estudio sobre UD-c vertebral y un estudio sobre UD-c carotídeo, por lo que no se pudo realizar análisis estadístico de estas 2 pruebas diagnósticas. EI UD-c Transcraneal fue reportado en 34 estudios, incluyendo 2,964 participantes (edad promedio de 46.8 años; $52 \%$ masculinos). Para el conjunto de estudios, se calculó una sensibilidad de $95 \%$ (IC 95\% de 0.93-0.97) y especificidad de 95\% (IC de $95 \%$ de 0.89-0.98); se calcularon además likelihood ratio (+) de 8.93 (IC de $95 \%$ de 7.17-11.0), un likelihood ratio (-) de 0.08 (IC de $95 \%$ de 0.05-0.12) y, al elaborar el nomograma de Bayes, infiriendo una probabilidad pretest de $30 \%$ (prevalencia de CDI en la población general), se calculó una probabilidad postest para su respectivo likelihood ratio (+) de $79 \%$ (IC $0.75-0.82$ ), y una probabilidad postest para su respectivo likelihood (-) de $3 \%$ (IC 0.02-0.05).

Conclusiones: EI UD-c transcraneal es una prueba con un excelente rendimiento diagnóstico al compararse contra el ETE-c, cuenta con la desventaja de no permitir diferenciar el tipo de comunicación (intracardiaca o pulmonar). No existe suficiente evidencia para recomendar el uso de UD-c vertebral o carotídeo para el diagnóstico de CDI; sin embargo, la evidencia actual orienta hacia una superioridad del UD-c transcraneal sobre éstos.

Palabras clave: Ultrasonido Doppler contrastado, ecocardiograma transesofágico contrastado, ultrasonido Doppler transcraneal, cortocircuito de derecha a izquierda.

\section{ABSTRACT}

Introduction: Right to left shunt (RLS) occurs mainly with intracardiac lesions such as patent foramen ovale and, to a lesser extent, through pulmonary arteriovenous malformations. Since the initial report by Gramiak et al., contrast echocardiography has been the method of choice to identify RLS. Being the Transesophageal Echocardiogram with contrast (TEE-c) currently accepted by many authors as the ideal diagnostic method of RLS.

Material and methods: A systematic review was carried out, with the objective of knowing the diagnostic performance of the contrast doppler ultrasound (DU-C) in its different variants (transcranial, vertebral and carotid) compared to TEE-c for the diagnosis of RLS; the review was carried out in the EBSCO, Elsevier, KoreaMed, Lilacs, PubMed/Medline, OVID/Cochrane Library, Scielo, SCOPUS, Web of Science and EMBASE databases.

Results: 33 publications were obtained that met the inclusion criteria, providing 36 studies with 3,099 participants (average age $47.1 ; 53 \%$ male). Of these, only 1 study on vertebral UD-c and 1 study on carotid UD-c were included, so it was not possible to perform statistical analysis of these 2 diagnostic tests.

\footnotetext{
* Médico residente de Medicina Crítica
}

¥ Médico adscrito.

Unidad de Terapia Intensiva «Alberto Villazón Sahagún», Hospital Español de México.

Recepción: 30/08/2019. Aceptación: 15/10/2019.

www.medigraphic.com/medicinacritica
Transcranial UD-c was reported in 34 studies, including 2,964 participants (average age 46.8 years, 52\% male); for the set of studies, a sensitivity of $95 \%(95 \% \mathrm{Cl}$ of $0.93-0.97)$ and specificity of $95 \%(95 \% \mathrm{Cl}$ of $0.89-0.98)$ were calculated; likelihood ratio (+) of $8.93(95 \% \mathrm{Cl}$ of 7.17-11.0), and a Likelihood ratio (-) of 0.08 (95\% Cl of 0.05-0.12) were also calculated, and when making Bayes' nomogram, inferring a probability Pre-Test of $30 \%$ (prevalence of CDI in general population), a post-test probability is calculated for their respective Likelihood ratio (+) of $79 \%$ (Cl 0.75-0.82), and a post-test probability for their respective likelihood (-) of 3\% (Cl 0.02-0.05).

Conclusions: The transcranial UD-c is a test with an excellent diagnostic performance when compared to ETE-C, it has the disadvantage of not allowing to differentiate the type of communication (intracardiac or pulmonary). There is not enough evidence to recommend the use of vertebral or carotid UD-C for the diagnosis of CDI; however, current evidence points to a superiority of transcranial UD-c over these.

Keywords: Contrasted Doppler ultrasound, contrasted transesophageal echocardiogram, transcranial Doppler ultrasound, right to left shunt.

\section{RESUMO}

Introdução: A comunicação da direita para a esquerda (CDE) ocorre principalmente com lesões intracardíacas, como forame oval permeável (FOP) e, em menor grau, por malformações arteriovenosas pulmonares (MAVP). Do relatório inicial de Gramiak et al. A ecocardiografia com contraste tem sido o método de escolha para identificar CDE. Sendo o ecocardiograma transesofágico com contraste (ETE-C) atualmente considerado o método diagnóstico ideal para $C D E$

Material e métodos: Foi realizada uma revisão sistemática, a fim de conhecer o desempenho diagnóstico do ultrassom Doppler por contraste (UD-c) em suas diferentes variantes (transcraniana, vertebral e carótida) em comparação ao TEE-c para o diagnóstico de CDE; a revisão foi realizada nas bases de dados EBSCO, Elsevier, KoreaMed, Lilacs, PubMed/Medline, OVID/Cochrane Library, Scielo, SCOPUS, Web of Science e EMBASE.

Resultados: Foram obtidas 33 publicações que atenderam aos critérios de inclusão, fornecendo 36 estudos com 3,099 participantes (idade média 47.1; $53 \%$ homens). Desses, apenas 1 estudo em UD-c vertebral e 1 em carotídeo UD-c foi incluído, portanto não foi possível realizar análise estatística desses dois testes de diagnóstico. UD-c transcraniana foi relatada em 34 estudos, incluindo 2,964 participantes (idade média de 46.8 anos, $52 \%$ do sexo masculino); para o conjunto de estudos cálculos-se uma sensibilidade de 95\% (IC95\% 0.93-0.97) e especificidade de 95\% (IC95\% 0.89-0.98); a razão de verossimilhança positiva de 8.93 (IC 95\% de 7.17-11.0) e uma razão de verossimilhança negativa de 0.08 (IC 95\% de 0.05-0.12) também foram calculadas e, ao elaborar o normograma de Bayes, inferir uma probabilidade Pré-teste de 30\% (prevalência de CDE na população em geral), uma probabilidade pós-teste é calculada para sua respectiva razão de verossimilhança positiva de $79 \%$ (IC 0.75-0.82) e uma probabilidade pósteste para suas respectivas probabilidade (-) de $3 \%$ (IC 0.02-0.05).

Conclusões: O UD-c é um teste com excelente desempenho diagnóstico quando comparado ao ETE-c, tem a desvantagem de não permitir diferenciar o tipo de comunicação (intracardíaca ou pulmonar). Não há evidências suficientes para recomendar o uso de UD-c vertebral ou carotídea para o diagnóstico de $C D E ;$ no entanto, as evidências atuais apontam para a superioridade da UD-C transcraniana em relação a elas.

Palavras-chave: Ultrassom Doppler contrastado, ecocardiograma transesofágico contrastado, ultrassom Doppler transcraniano, derivação da direita para a esquerda.

\section{INTRODUCCIÓN}

La comunicación de derecha a izquierda (CDI), producida principalmente por lesiones intracardiacas, como el fora- 
men oval permeable (FOP) y, en menor medida, a través de malformaciones arteriovenosas pulmonares (MAVP), se ha asociado con una variedad de procesos patológicos comunes como migraña y eventos vasculares cerebrales. ${ }^{1}$

Desde el informe inicial de Gramiak y otros, ${ }^{2}$ la ecocardiografía con contraste salino ha sido el método de elección para identificar la CDI. Al agitar una pequeña cantidad de aire con soluciones, tales como solución salina a $0.9 \%$ o dextrosa a $5 \%$, se producen microvesículas o «burbujas» que, cuando se inyectan en la circulación venosa, aparecen en el lado izquierdo del corazón únicamente cuando existe una CDI. Ésta, por tanto, se ha propuesto como un criterio de diagnóstico para identificar la derivación hacia la izquierda.

Por un lado, el ecocardiograma transesofágico (ETT) es el punto de partida habitual; sin embargo, algunos pacientes pueden requerir un ecocardiograma transesofágico con contraste (ETE-c) para la detección de CDI. Por el otro, el ultrasonido Doppler contrastado (UD-c) puede identificar CDI, pero no puede especificar su ubicación; la presencia de microvesículas en la circulación arterial de grandes vasos de cabeza y cuello (arterias carotídeas, vertebrales o transcraneales) se considera diagnóstica de CDI. En general, la «aparición temprana» de microvesículas en el lado izquierdo del corazón (dentro de los tres latidos de la opacificación del corazón del lado derecho) se considera indicativa de una derivación intracardiaca, mientras que las burbujas que aparecen más tarde se consideran sugestivas de una CDI extracardiaca (pulmonar). ${ }^{1}$

La causa más común de CDI es el FOP. Éste es un componente obligado de la circulación fetal, que se cierra por fusión del septum primum y del septum secundum poco después del nacimiento. Aunque esta comunicación se cierra en la mayoría, sigue persistente en aproximadamente $30 \%$ de los individuos. Los estados de enfermedad más comúnmente asociados con FOP incluyen evento vascular cerebral criptogénico y migraña. La prevalencia de la derivación extracardiaca a través de la MAVP en la población general no está bien estudiada, y se considera que su presencia es poco frecuente, ${ }^{1}$ reportándose incidencias en individuos sanos de hasta $28 \%$, y en $5 \%$ de los casos se ha reportado MAVP y FOP simultáneamente ${ }^{3}$ asociándose más estrechamente su presencia con patologías y trastornos vasculares, como la telangiectasia hemorrágica hereditaria y la enfermedad hepática avanzada. ${ }^{1}$

Los agentes de contraste ultrasonográficos son sustancias libres de radiación que mejoran la claridad de la imagen del ultrasonido. A menudo, se utilizan para «rescatar» ecografías «técnicamente difíciles», por ejemplo, cuando la transmisión de señales del ultrasonido se ve afectada por la obesidad u otros impedimentos físicos. ${ }^{4}$

La detección de FOP mediante ETE-c es el método de elección como técnica no invasiva, cuya alta confiabilidad ha sido demostrada mediante autopsia y cateterización cardiaca. La ETT convencional generalmente falla porque la maniobra de Valsalva, que es necesaria para crear la derivación de derecha a izquierda, conduce a una disminución en la calidad de la imagen. ${ }^{5}$

EI UD-c, particularmente el ultrasonido Doppler transcraneal con contraste, se ha utilizado ampliamente para la detección de CDI, ya que es un instrumento confiable y reproducible en comparación con el ETE-c. Sin embargo, la detección de CDI está limitada por ventanas de hueso temporal insuficientes en 10 a $20 \%$ de los pacientes. ${ }^{6}$ Las imágenes de ultrasonido con contraste de la arteria carótida común con maniobra de Valsalva podrían ser una herramienta de detección opcional para la detección del CDI en pacientes con ventanas óseas acústicas insuficientes. ${ }^{7,8}$ De la misma manera, el UD-c vertebrobasilar también se ha considerado y comparado contra UD-c transcraneal para detectar CDI. ${ }^{6}$

\section{MATERIAL Y MÉTODOS}

Se realizó una revisión sistemática, cuyo objetivo es conocer el rendimiento diagnóstico del ultrasonido Doppler contrastado (UD-c) en sus diferentes variantes (transcraneal, vertebral y carotídeo) en comparación con el ETE-c para el diagnóstico de CDI. Se incluyeron para tal fin artículos prospectivos que evaluaran el rendimiento diagnóstico del UD-c contra el ETE-c, y que reportaran los datos suficientes para poder realizar una tabla de $2 \times 2$ y calcular verdaderos positivos (VP), verdaderos negativos (VN), falsos positivos (FP) y falsos negativos (FN) de UD-c en relación con ETE-c. Se excluyeron estudios realizados en población pediátrica y estudios que utilizaran un método diagnóstico de comparación diferente a ETE-c.

De esta manera, se realizó una búsqueda de artículos en las bases de datos EBSCO, Elsevier, KoreaMed, Lilacs, PubMed/Medline, OVID/Cochrane Library, Scielo, SCOPUS, Web of Science y EMBASE. La búsqueda se completó en julio de 2019, sin límite inferior para la búsqueda. Los términos usados en la búsqueda fueron los siguientes: «right-to-left shunt» or «right-to-left shunting» or «right to left shunt» or «right to left shunting» and («contrast-enhanced transcranial Doppler» or "contrast-enhanced vertebral Doppler» or «contrast-enhanced carotid Doppler» or «contrastenhanced transcranial sonography» or «contrast-enhanced vertebral sonography» or "contrast-enhanced carotid sonography» or "contrast-enhanced transcranial» or «contrast-enhanced vertebral» or «contrastenhanced carotid» or "extracranial duplex sonography» or "extracranial sonography» or "carotid artery monitoring" or "vertebral artery monitoring» or «middle cerebral artery monitoring»). 
Después de realizar la búsqueda de los términos, se analizaron las referencias de todos los estudios primarios, así como de las revisiones conocidas, para hallar los estudios citados que no se encontraron en la búsqueda inicial. No se utilizaron restricciones con respecto al idioma de publicación.

Los datos se extrajeron en una hoja de cálculo con información sobre el diseño del estudio, el tipo de UD-c empleado, el tamaño de la cohorte, la edad, el sexo, el tipo de contraste utilizado, el método de provocación y duración, el corte de microburbujas utilizado para un estudio UD-c positivo y los resultados de precisión de prueba (VP, VN, FP y FN). Se excluyeron los estudios que no utilizaran el ETE-c como estándar de oro para el diagnóstico de CDI. Dos revisores (MFGT y SAVM) evaluaron de forma independiente los artículos, y las discrepancias se resolvieron por consenso. ${ }^{9}$

Utilizando el programa RStudio ${ }^{\circledR}$ versión $1.1 .463^{\odot}$ se tabularon los VP, VN, FP y FN de cada estudio, se realizó el cálculo de sensibilidad y especificidad, y se produjo la gráfica de forest plot con los datos obtenidos. Pos- teriormente, se construyó la curva de ROC y se calculó el área bajo la curva para interpretar el rendimiento diagnóstico de la prueba. También se calcularon la probabilidades pretest y postest de la prueba, utilizando el nomograma de Bayes, con un punto de inicio de $30 \%$, prevalencia promedio de foramen oval permeable (principal causa de CDI) en la población sana. Asimismo, utilizando el programa RevMan, se calculó el riesgo de sesgo de forma independiente para cada estudio según la escala para evaluación del riesgo de sesgo QUADAS II, y se graficó la totalidad de los estudios.

\section{RESULTADOS}

Se encontraron 174 artículos según los criterios de búsqueda (al respecto, la Figura 1 muestra el diagrama de flujo para la selección de artículos); de éstos, se eliminaron 137 por revisión de título y resumen, y los 37 artículos restantes pasaron a revisión en detalle, con revisión de estudios en referencias cuando estas sugerían posibilidad de extender la muestra de estudio. Se encontró

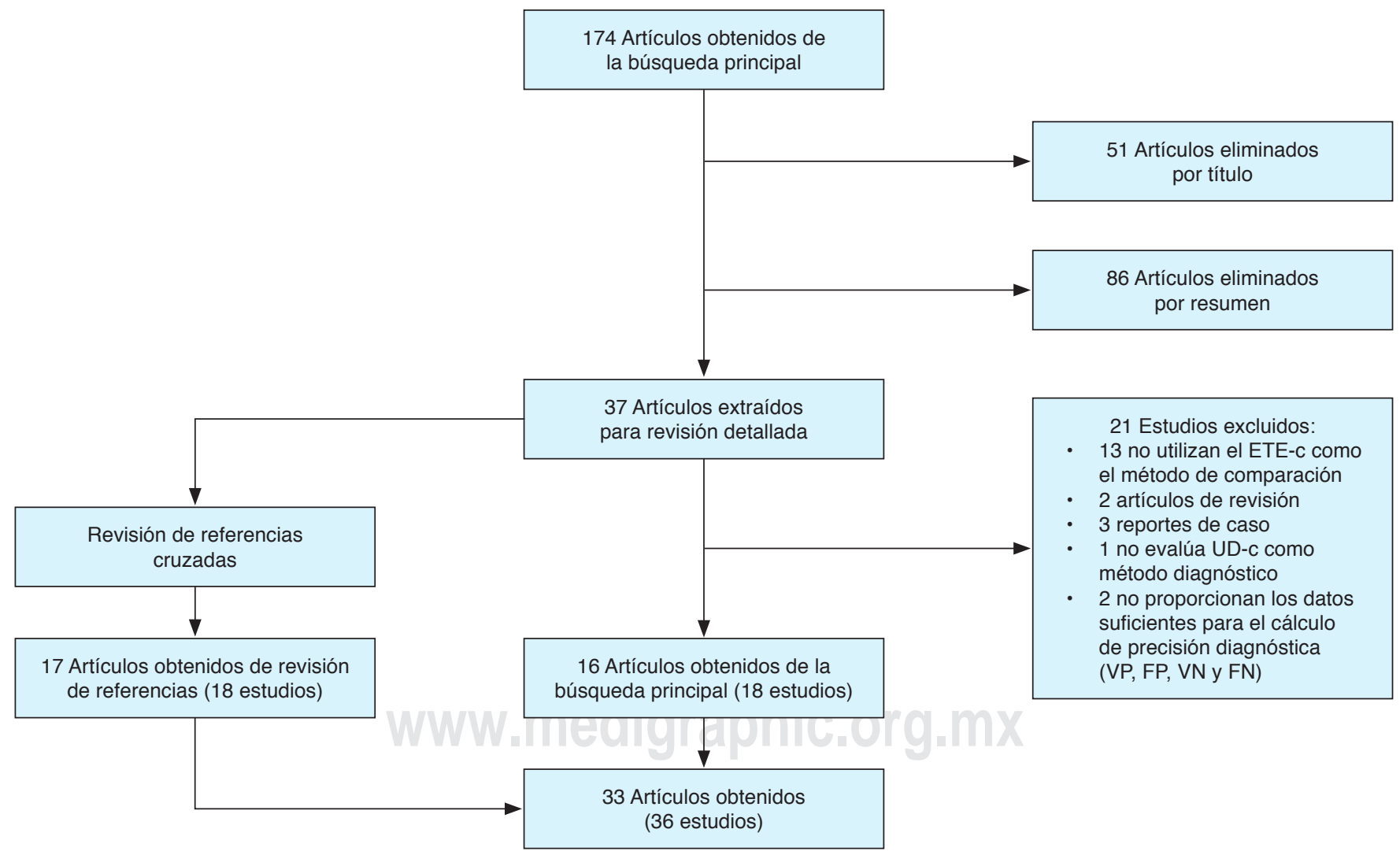

Se obtuvieron 174 artículos en la búsqueda inicial, de donde se descartaron 137 por revisión de título y resumen; 37 artículos fueron aprobados para revisión detallada a través de la revisión de las referencias presentes cuando el autor lo consideró apropiado. Se excluyeron 21 artículos por no cumplir con los criterios de inclusión y, de esta manera, se obtuvieron 16 artículos de la búsqueda principal para incluir en el metaanálisis. Asimismo, se incluyeron 17 artículos obtenidos por la revisión de referencias; obteniendo un total de 33 artículos, con 36 estudios realizados, incluibles en el metaanálisis.

$\mathrm{ETE}-\mathrm{c}=$ ecocardiograma transesofágico con contraste, UD-c $=$ ultrasonido Doppler contrastado, $\mathrm{VP}=$ verdaderos positivos, $\mathrm{FP}=$ falsos positivos, $\mathrm{VN}=$ verdaderos negativos, $\mathrm{FP}=$ falsos negativos.

Figura 1: Flujograma de selección de estudios. 
Tabla 1: Características de los estudios incluidos.

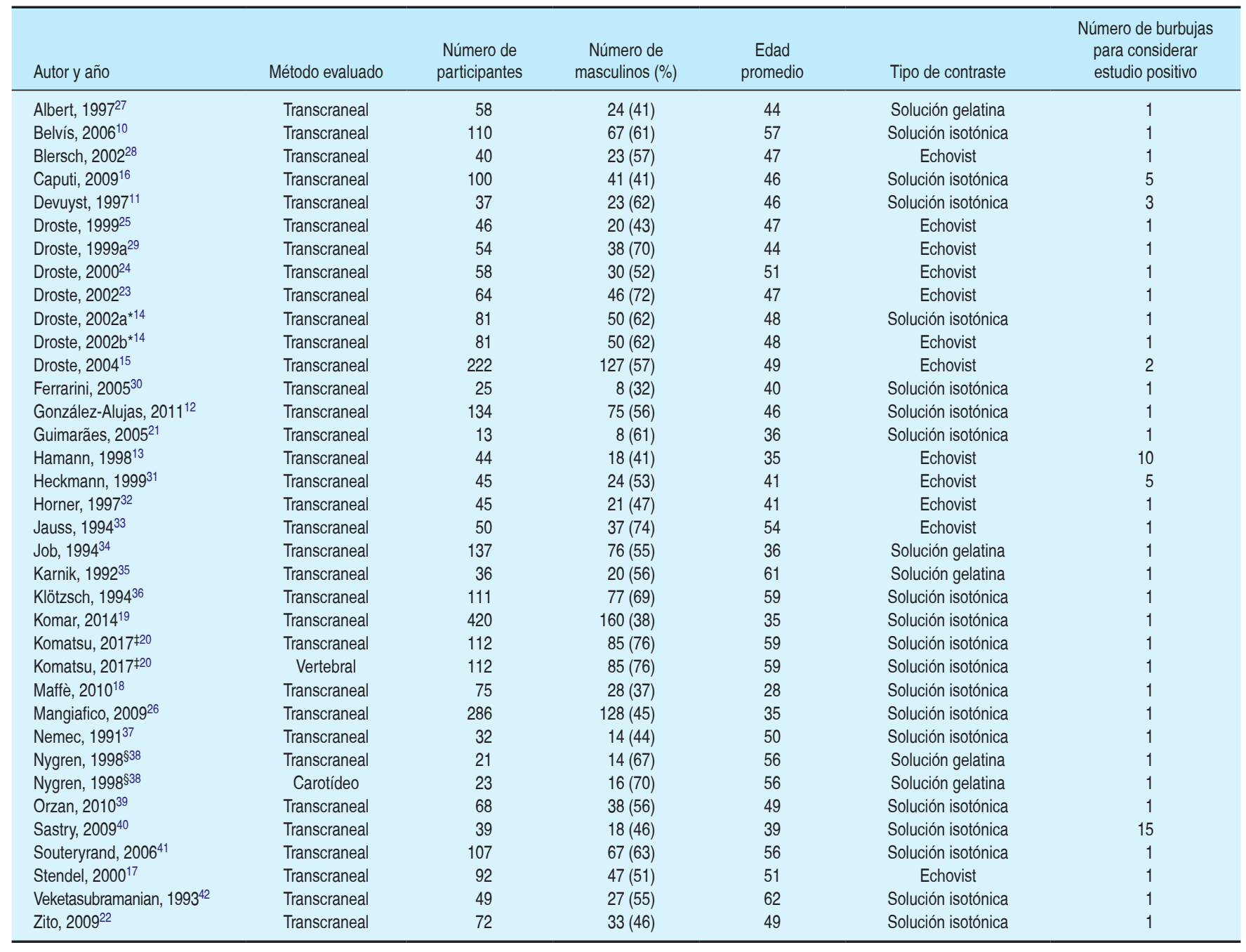

*Se realizó en el mismo estudio evaluación de UD-c transcraneal con dos medios de contraste diferentes, ambos comparados con ETE-c de forma independiente. ₹Se realizó en el mismo estudio evaluación de UD-c transcraneal y vertebral, ambos comparados contra ETE-c de forma independiente. § Se realizó en el mismo estudio evaluación de UD-c transcraneal y carotídeo, ambos comparados contra ETE-c de forma independiente. NE = no especificado; UD-c = ultrasonido Doppler contrastado; ETE-C = ecocardiograma transesofágico con contraste. Maniobra de provocación Valsalva.

además con esta búsqueda un metaanálisis de Mojadidi y otros, ${ }^{43}$ de donde se tomaron todas las referencias citadas que se asociaron con la evaluación de UD-c, así como de las demás revisiones asociadas con UD-c encontradas en la búsqueda original. De esta manera, se excluyeron 21 artículos de la búsqueda original por no cumplir con los criterios de inclusión para obtener16 artículos, uno de los cuales presentaba información referente a dos estudios diferentes de UD-c transcraneal (comparación de UD-c transcraneal con diferentes métodos de contraste contra ETE-c) ${ }^{14}$ y otro presentaba evaluación de forma independiente entre UD-c transcraneal contra ETE-c y UD-c vertebral contra ETE-c, obteniéndose finalmente 18 estudios que cumplían con los criterios de inclusión para el análisis estadístico.

Tras completar la revisión de los estudios obtenidos en las referencias de los trabajos de revisión asociados con el tema, se logró la inclusión de 17 artículos más: uno de éstos presentaba una evaluación de forma independiente entre UD-c transcraneal contra ETE-c, y UD-c carotídeo contra ETE-c. De esta manera se obtuvieron 18 estudios más, completándose finalmente una muestra total de 33 artículos con 36 estudios candidatos para realizarse análisis estadístico. En la Tabla 1 se recopilan las características de los estudios, obteniéndose una muestra de 36 estudios, con 3,099 participantes (edad promedio de $47.1 \pm 17$ años; $53 \%$ del sexo masculino).

Se realizó evaluación del riesgo de sesgo con la herramienta QUADAS II (Figura 2), la cual, mayoritariamente, presenta datos de bajo riesgo de sesgo. No obstante, dicha herramienta presenta, como característica común, un alto riesgo de sesgo en el apartado de selección de pacientes, lo cual es determinado por 
el autor, debido a que la población estudiada siempre constaba con el antecedente de patología asociada con $\mathrm{CDI}$, por lo que en ninguno de éstos contaba con grupo control de pacientes sanos (puede que porque la probabilidad de diagnóstico de CDI fuera mayor que en la población general).

De los estudios que cumplieron con los criterios de inclusión, un estudio evaluó el UD-c vertebral y otro estudio el UD-c carotídeo, por lo que no se pudo realizar análisis estadístico de estas dos pruebas diagnósticas, imposibilitándose su evaluación con metaanálisis.

EI UD-c transcraneal fue reportado en 34 estudios (de los 33 artículos) con una muestra de 2,964 participantes (siendo $52 \%$ del sexo masculinos), una media de edad de $46.8 \pm 17$ años. Para el conjunto de estudios, se calculó una sensibilidad de 95\% (IC de 95\% de $0.93-0.97$ ) con $\mathrm{I}^{2}$ de $67 \%$, y especificidad de $95 \%$ (IC de $95 \%$ de $0.89-0.98$ ) con $\mathrm{I}^{2}$ de $93 \%$ (Figuras 3 y
4). Con estos estudios, se elaboró la curva de ROC, apreciándose una excelente distribución de la mayor parte, con un área bajo la curva de $96 \%$ (Figura 5); se calcularon, además, likelihood ratio (+) de 8.93 (IC de $95 \%$ de $7.17-11.0)$, un likelihood ratio (-) de 0.08 (IC de 95\% de 0.05-0.12) y, al elaborar el nomograma de Bayes, infiriendo una probabilidad pretest de $30 \%$ (prevalencia de CDI en la población general). Se calculó una probabilidad postest de 79\% (IC 0.75-0.82) cuando la prueba fue positiva y una probabilidad postest de $3 \%$ (IC 0.02-0.05) cuando la prueba fue negativa (Figura 6).

Respecto al medio de contraste, algunos estudios reportaron que no existe una diferencia significativa en la sensibilidad entre ambos materiales. En la presente revisión, 54\% de los artículos reporta la realización de sus estudios con solución salina isotónica agitada como medio de contraste; $34 \%$ reporta uso de medio

A)

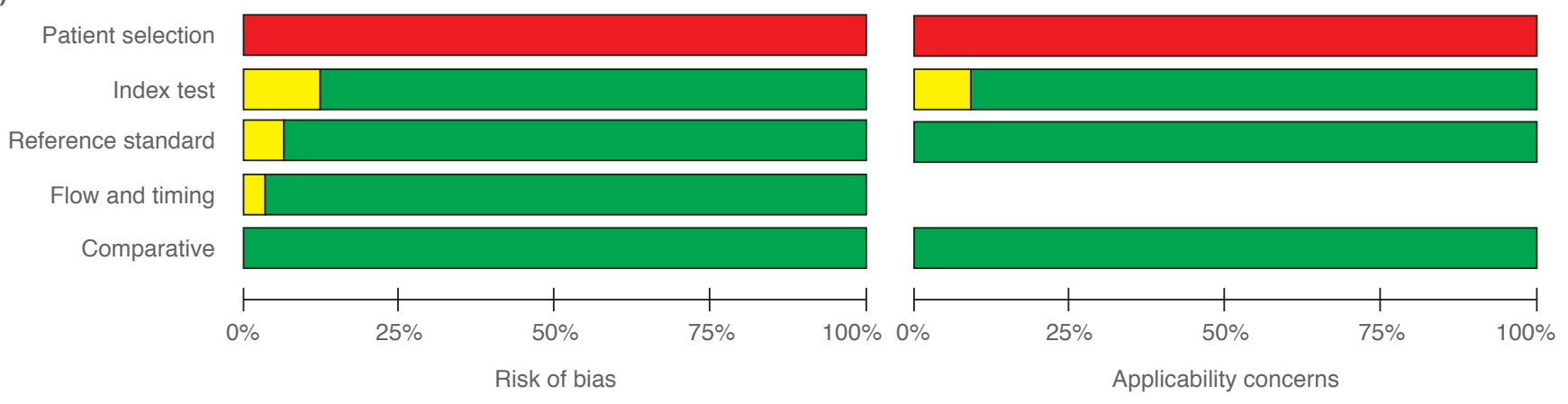

B)

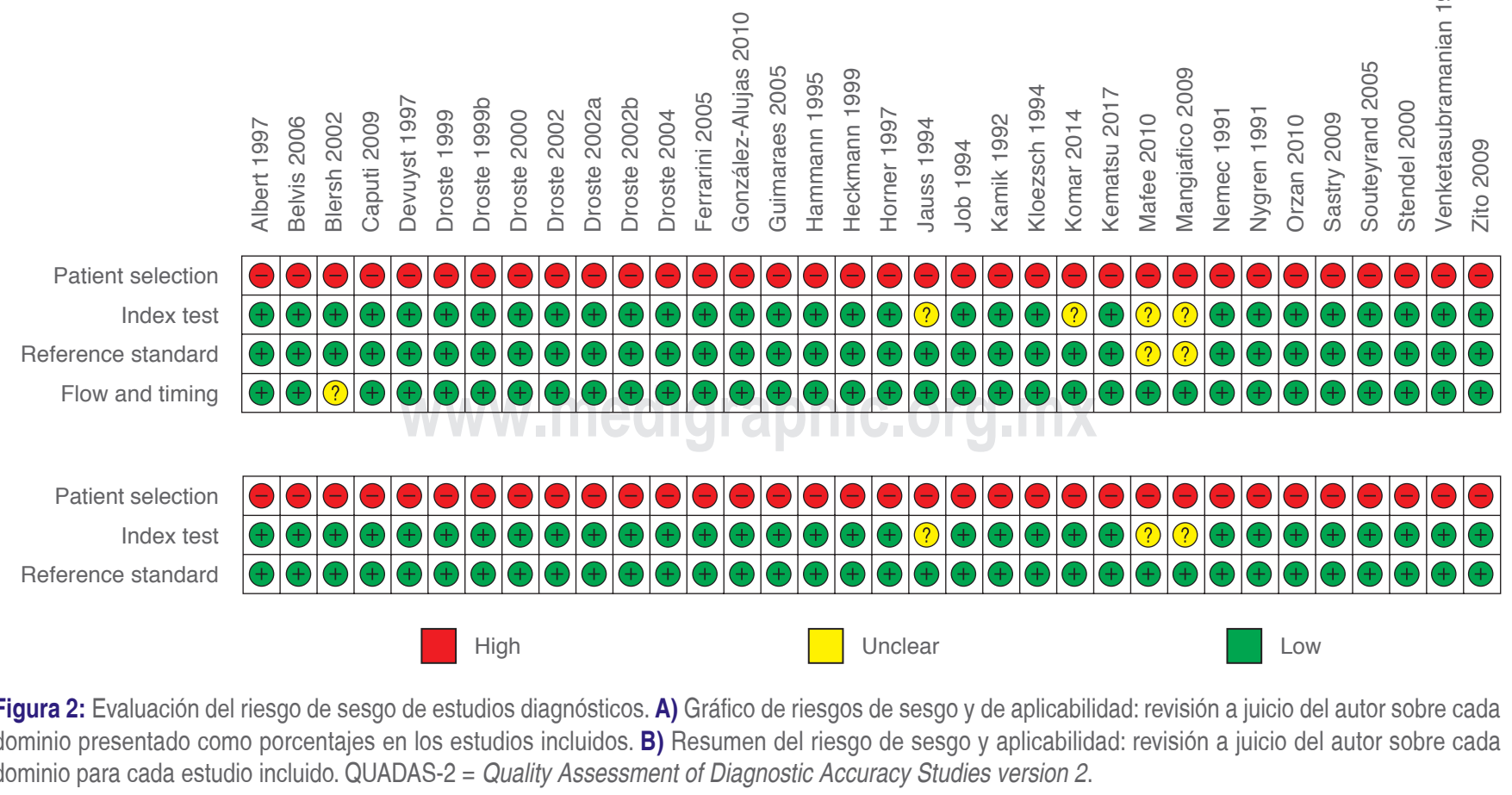


Figura 3: Gráfico de forest plot para la sensibilidad.

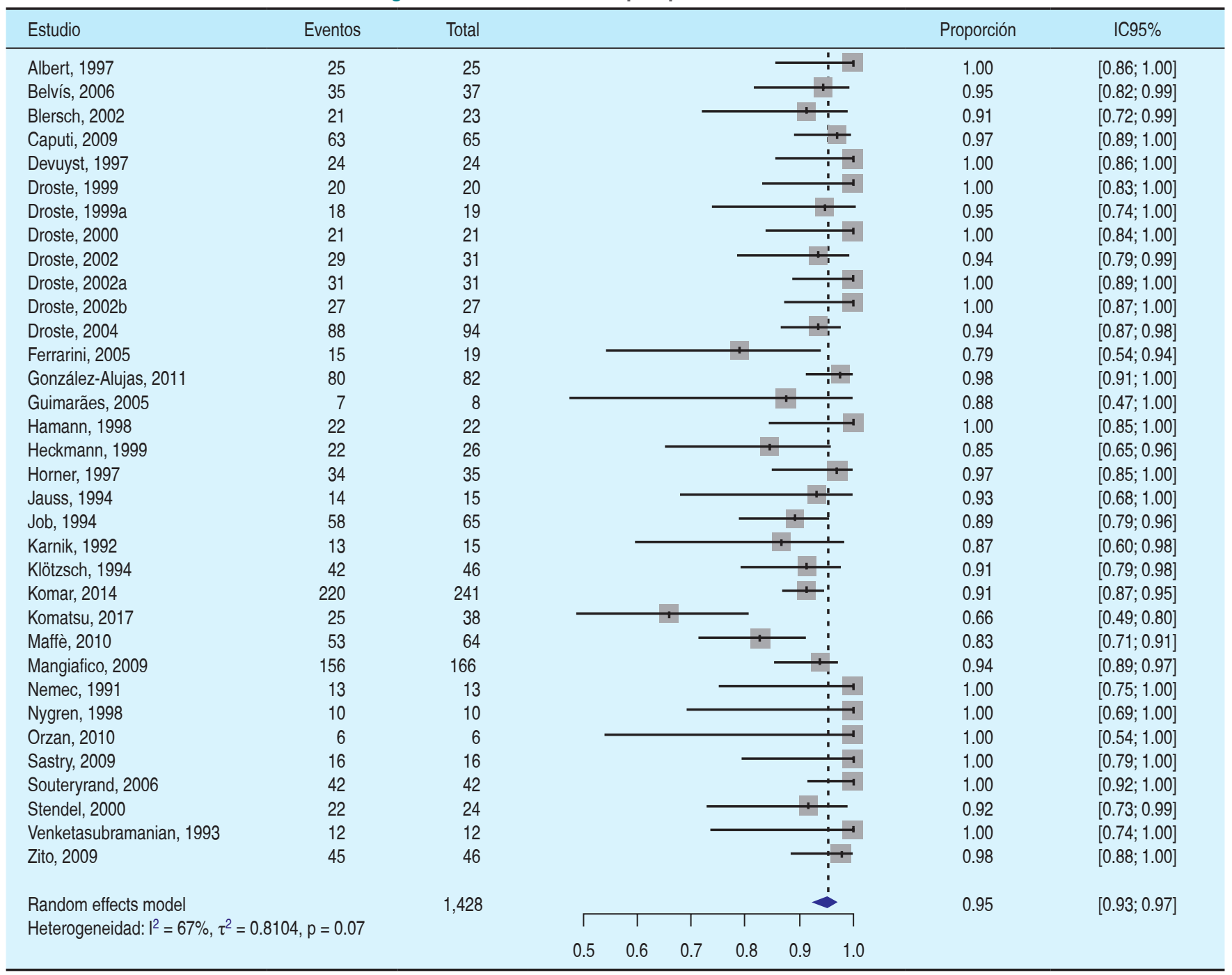

de contraste Echovist, y $11 \%$ reporta uso de soluciones coloides (gelatina) para sus estudios (sensibilidades de 94, 95 y 94\%, respectivamente, no representando diferencia alguna). Sólo un artículo comparó el uso de UD-c transcraneal con diferentes medios de contraste (solución isotónica contra Echovist), reportándose sensibilidades de 94 y $100 \%$, respectivamente, sin mostrar una diferencia estadísticamente significativa, pero recomendando el uso de Echovist cuando la prueba con solución isotónica resulte negativa con alta sospecha de CDI. ${ }^{14}$

\section{DISCUSIÓN}

Únicamente se encontraron dos estudios que compararan el UD-c vertebral y carotídeo (un estudio de cada uno). Estas dos investigaciones evaluaron la precisión diagnóstica de cada uno contra ETE-c, reportando una mala sensibilidad (53 y $58 \%$, respectivamente). Ade- más, en los mismos estudios, se comparó contra UD-c transcraneal, los cuales reportaron mejor sensibilidad que éstos (65 y 100\% respectivamente); sin embargo, la especificidad reportada fue buena para UD-c vertebral y carotídeo (100 y $91 \%$, respectivamente). Cabe señalar que no se pudo realizar análisis estadístico, debido a que no se encontraron más estudios. ${ }^{20,38}$

De forma individual para el UD-c transcraneal, se reportó una excelente sensibilidad y especificidad para el conjunto de estudios analizados con valores de 95\% para cada uno; sin embargo, es importante destacar que los valores de $\mathrm{I}^{2}$ fueron de 67 y $93 \%$ respectivamente, reflejando una moderada sensibilidad para el metaanálisis de sensibilidad, pero reflejando una alta heterogeneidad para el metaanálisis de especificidad, lo cual plantea dudas en la veracidad con la que se debe tomar este resultado, y es concordante con la gran variabilidad en los valores de especificidad reportados en los estudios. 
Las maniobras de provocación durante la realización de UD-c transcraneal se han estandarizado como parte indispensable del procedimiento, lo cual en múltiples estudios se ha demostrado cómo afecta de forma significativa su sensibilidad; en el presente análisis, únicamente se incluyeron los estudios en los cuales se realizó maniobra de provocación, siendo la maniobra de Valsalva la que se utilizó en la totalidad de éstos. La duración del tiempo de la maniobra de provocación es otro punto de debate, pues se ha referido como apropiado un valor en torno a 10 segundos; en la presente revisión, se encontró que sólo $20 \%$ la realizó con una maniobra mínima de 10 segundos, en tanto que en la mayoría (38\%), se usó una maniobra de sólo cinco segundos (35\% de los estudios reporta el uso de maniobra de provocación, pero no la duración de la misma). ${ }^{23}$

Cabe destacar que, al utilizar el ETE-c como estándar de referencia, se está valorando la sensibilidad y especificidad del UD-c para el diagnóstico de CDI intracardiaca, es decir, el CDI de origen pulmonar difícilmente puede ser evidenciado mediante ETE-c, y constituye un potencial condicionante de resultados falsos negativos para el UD-c. Llama la atención, inclusive, que algunos autores refieren que el ETE-c puede tener menor sensibilidad diagnóstica que UD-c para CDI de cualquier causa. ${ }^{10,19,31,32}$

Es de especial interés para los médicos en la Unidad de Terapia Intensiva el efecto de la maniobra de Valsalva sobre los cortocircuitos, demostrando que un defecto anatómico que condicione cortocircuito no necesariamente condicionará un flujo sanguíneo de derecha a izquierda. Debe incluso aclarase que el cortocircuito más comúnmente tendrá una dirección de izquierda a derecha por las presiones más elevadas que se manejan en las cavidades izquierdas del corazón, creándose la CDI sólo cuando se ejerce una presión positiva pulmonar con la maniobra de

Figura 4: Gráfico de forest plot para especificidad.

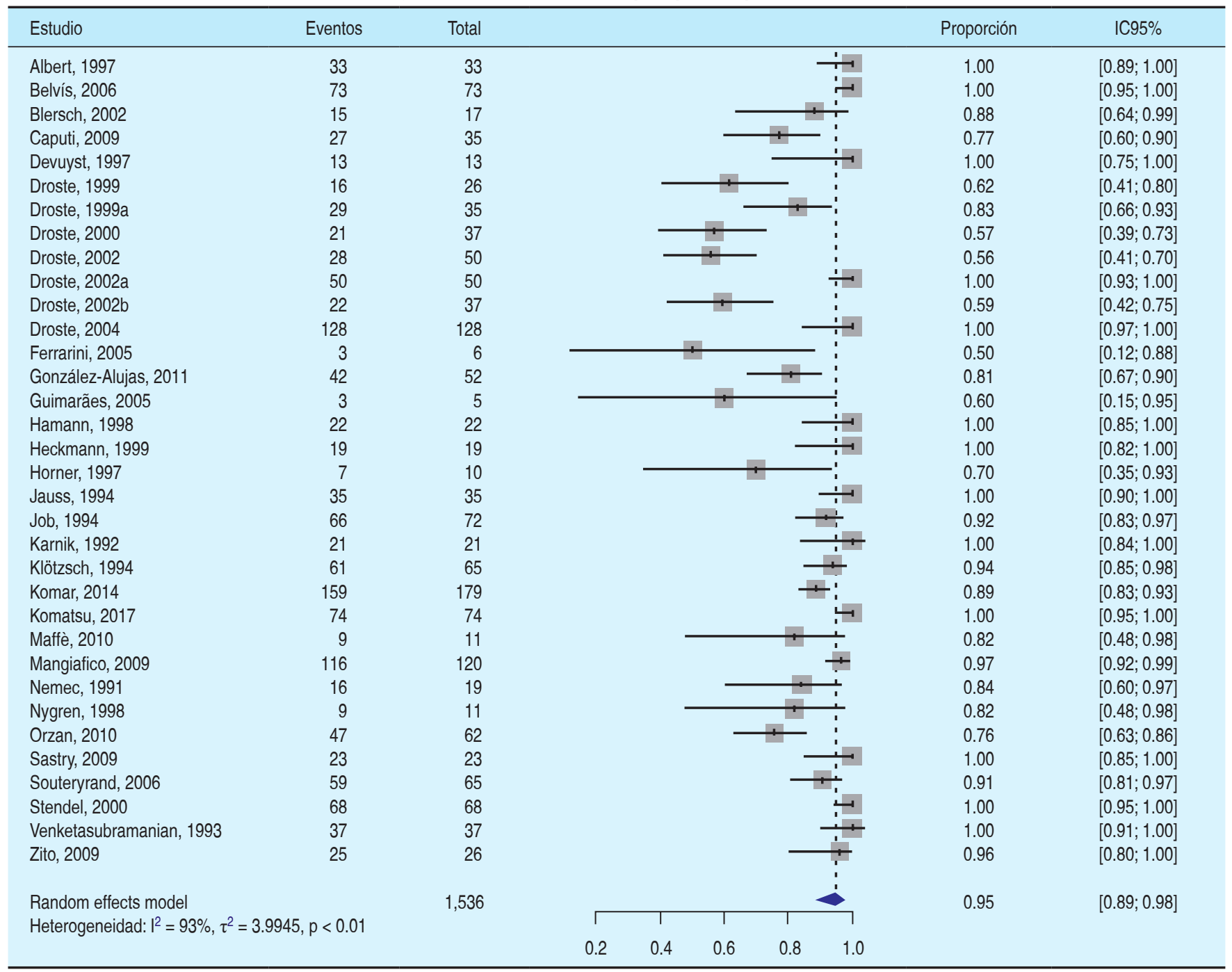


Valsalva. Esto supone que los pacientes con ventilación mecánica con presión positiva tendrán una mayor predilección a presentar CDI que no era existente o manifiesta, previo a que se realizara la aplicación de presión positiva en la vía aérea, y que pudiese, por ende, producir cambios clínicos o gasométricos en pacientes sin previa manifestación de patología respiratoria o hemodinámica; esto supone que sería importante sospechar y diagnosticar esta patología de forma habitual en los pacientes con ventilación mecánica invasiva y falla respiratoria sin causa establecida.

Cabe resaltar que no se encontró ningún estudio en el cual se reportara la evaluación diagnóstica con UD-c en pacientes con ventilación mecánica invasiva, lo cual supone un área de oportunidad para futuros ensayos clínicos.

\section{CONCLUSIONES}

EI UD-c transcraneal es una prueba con un excelente rendimiento diagnóstico al compararse con el ETE-c. Tiene como ventaja que es un estudio menos invasivo y que se realiza con mayor facilidad, pero cuenta con la desventaja de que no permite diferenciar el tipo de comunicación intracardiaca responsable de la CDI ni permite diferenciar entre CDI de origen intracardiaca o intrapulmonar.

Consideramos que es necesario realizar estudios con un método de referencia distinto que defina la

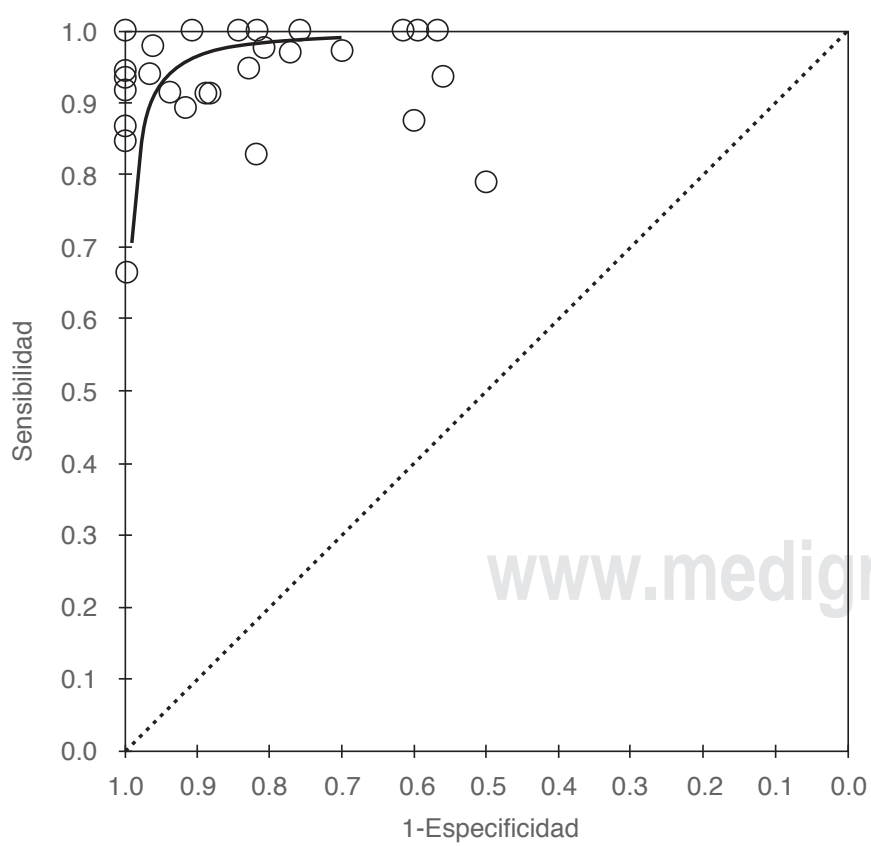

Curva característica operativa de receptor (ROC, por sus siglas en inglés) para los 34 estudios incluidos en el metaanálisis, elaborada con un cálculo de sensibilidad de $95 \%$ y especificidad de $95 \%$. Se calculó un área bajo la curva de $97 \%$.

Figura 5: Curva ROC.

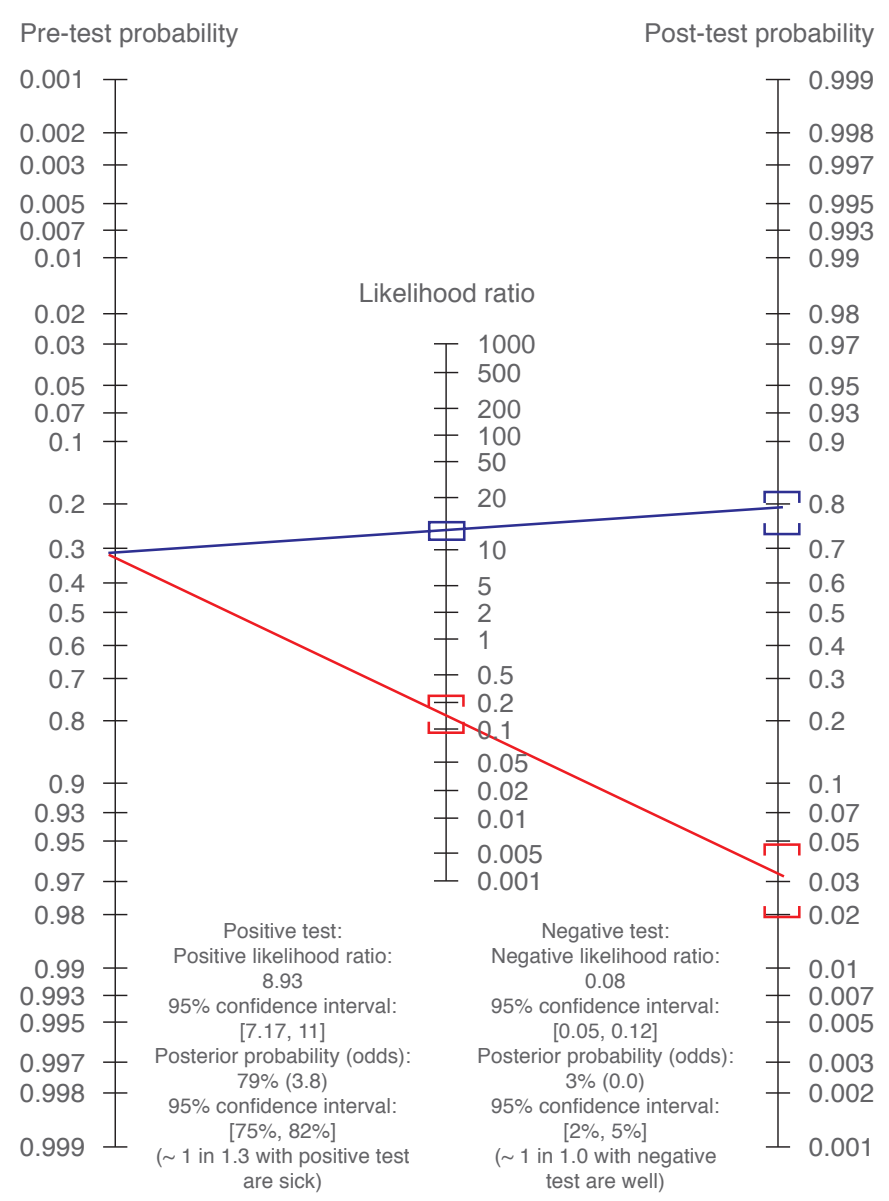

Figura 6: Probabilidades pre-test y post-test para CDI tras realización de ultrasonido Doppler transcraneal. Probabilidad predefinida de una población seleccionada, y probabilidad asociada posterior a la realización de la prueba, para un resultado positivo, y un resultado negativo. $\mathrm{CDI}=$ comunicación de derecha a izquierda.

precisión para el diagnóstico de CDI de origen intrapulmonar. El tipo de medio de contraste utilizado y la duración de la maniobra de provocación no parecen tener un impacto significativo en la precisión del UD-c. No existe suficiente evidencia como para recomendar el uso de UD-c vertebral o carotídeo para el diagnóstico de CDI; sin embargo, la evidencia actual orienta hacia una superioridad del UD-c transcraneal sobre estos dos.

\section{BIBLIOGRAFÍA}

1. Tighe D, Aurigemma G. Right-to-left shunts and saline contrast echocardiography. Chest. 2010;138:246-248.

2. Gramiak R, Shah P, Kramer D. Ultrasound cardiography: contrast studies in anatomy and function. Radiology. 1969;92:939-948.

3. Woods T, Harmann L, Purath T, Ramamurthy S, Subramanian S, Jackson S, et al. Small- and moderate-size right-to-left shunts identified by saline contrast echocardiography are normal and unrelated to migraine headache. Chest. 2010;138:264-269.

4. Parker J, Weller M, Feinstein L, Adams R, Main M, Grayburn M, et al. Safety of ultrasound contrast agents in patients with known 
or suspected cardiac shunts. Am J Cardiol. 2013;112:10391045.

5. Van H, Poommipanit P, Shalaby M, Gevorgyan R, Tseng $\mathrm{CH}$, Tobis J. Sensitivity of transcranial doppler versus intracardiac echocardiography in the detection of right-to-left shunt. JACC Cardiovasc Imaging. 2010;3:343-348.

6. Guo Y, Gao Y, Guo Z, Niu P, Yang Y, Xing Y. Comparison of vertebral artery and middle cerebral artery monitoring for right-toleft shunt detection by contrast-enhanced transcranial Doppler. Sci Rep. 2016;6:24932.

7. Draganski B, Blersch W, Holmer S, Koch H, May A, Bogdahn $U$, et al. Detection of cardiac right-to-left shunts by contrastenhanced harmonic carotid duplex sonography. J Ultrasound Med. 2005;24:1071-1076.

8. Topçuoglu MA, Palacios IF, Buonanno FS. Contrast M-mode power Doppler ultrasound in the detection of right-to-left shunts: utility of submandibular internal carotid artery recording. $J$ Neuroimaging. 2003;13:315-323.

9. Whiting PF, Rutjes AW, Westwood ME, Mallett S, Deeks JJ, Reitsma JB, et al. QUADAS-2: a revised tool for the quality assessment of diagnostic accuracy studies. Ann Intern Med. 2011;155:529-536.

10. Belvís R, Leta RG, Martí-Fàbregas J, Cocho D, Carreras F, Pons-Lladó $\mathrm{G}$, et al. Almost perfect concordance between simultaneous transcranial doppler and transesophageal echocardiography in the quantification of right-to-left shunts. $J$ Neuroimaging. 2006;16:133-138.

11. Devuyst G, Despland PA, Bogousslavsky J, Jeanrenaud $X$. Complementarity of contrast transcranial Doppler and contrast transesophageal echocardiography for the detection of patent foramen ovale in stroke patients. Eur Neurol. 1997;38:21-25

12. González-Alujas T, Evangelista A, Santamarina E, Rubiera M, Gómez-Bosch Z, Rodríguez-Palomares JF, et al. Diagnosis and quantification of patent foramen ovale. Which is the reference technique? Simultaneous study with transcranial Doppler, transthoracic and transesophageal echocardiography. Rev Esp Cardiol. 2011;64:133-139.

13. Hamann GF, Schätzer-Klotz D, Fröhlig G, Strittmatter M, Jost V, Berg G, et al. Femoral injection of echo contrast medium may increase the sensitivity of testing for a patent foramen ovale. Neurology. 1998;50:1423-1428.

14. Droste DW, Lakemeier S, Wichter T, Stypmann J, Dittrich R, Ritter M. Optimizing the technique of contrast transcranial Doppler ultrasound in the detection of right-to-left shunts. Stroke. 2002;33:2211-2216.

15. Droste DW, Schmidt-Rimpler C, Wichter T, Dittrich R, Ritter M, Stypmann J, et al. Right-to-left-shunts detected by transesophageal echocardiography and transcranial Doppler sonography. Cerebrovasc. 2004;17:191-196.

16. Caputi L, Carriero MR, Falcone C, Parati E, Piotti P, Materazzo C, et al. Transcranial Doppler and transesophageal echocardiography: comparison of both techniques and prospective clinical relevance of transcranial Doppler in patent foramen ovale detection. J Stroke Cerebrovasc Dis. 2009;18:343348.

17. Stendel R, Gramm HJ, Schröder K, Lober C, Brock M. Transcranial Doppler ultrasonography as a screening technique for detection of a patent foramen ovale before surgery in the sitting position. Anesthesiology. 2000;93:971-975.

18. Maffè S, Dellavesa $P$, Zenone F, Paino AM, Paffoni P, Perucca A, et al. Transthoracic second harmonic two- and three-dimensional echocardiography for detection of patent foramen ovale. Eur $J$ Echocardiogr. 2010;11:57-63.

19. Komar M, Olszowska M, Przewłocki T, Podolec J, Stępniewski $\mathrm{J}$, Sobień B, et al. Transcranial Doppler ultrasonography should it be the first choice for persistent foramen ovale screening? Cardiovasc Ultrasound. 2014;12:16-22.

20. Komatsu T, Terasawa Y, Arai A, Sakuta K, Mitsumura H, Iguchi Y. Transcranial color-coded sonography of vertebral artery for diagnosis of right-to-left shunts. J Neurol Sci. 2017;376:97-101.

21. Guimarães J, Santos R, Sousa G, De Oliveira P, Azebedo E. Standardized method for the detection of patent foramen ovale using transcranial colour-coded sonography, and comparison of the results with transesophageal echocardography. Sinapse. 2005;5:21-24.

22. Zito C, Dattilo G, Oreto G, Di Bella G, Lamari A, Iudicello R, et al. Patent foramen ovale: comparison among diagnostic strategies in cryptogenic stroke and migraine. Echocardiography. 2009;26:495-503.

23. Droste DW, Jekentaite R, Stypmann J, Grude M, Hansberg T, Ritter M, et al. Contrast transcranial Doppler ultrasound in the detection of right-to-left shunts: comparison of Echovist(R)-200 and Echovist $(\mathrm{R})-300$, timing of the Valsalva Maneuver, and general recommendations for the performance of the test. Cerebrovasc Dis. 2002;13:235-241.

24. Droste DW, Silling K, Stypmann J, Grude M, Kemény V, Wichter $\mathrm{T}$, et al. Contrast transcranial Doppler ultrasound in the detection of right-to-left shunts: time window and threshold in microbubble numbers. Stroke. 2000;31:1640-1645.

25. Droste DW, Kriete JU, Stypmann J, Castrucci M, Wichter T, Tietje $\mathrm{R}$, et al. Contrast transcranial Doppler ultrasound in the detection of right-to-left shunts: comparison of different procedures and different contrast agents. Stroke. 1999;30:1827-1832.

26. Mangiafico S, Scandura S, Ussia GP, Privitera A, Capodanno $D$, Petralia A, et al. Transesophageal echocardiography and transcranial color Doppler: independent or complementary diagnostic tests for cardiologists in the detection of patent foramen ovale? J Cardiovasc Med. 2009;10:143-148.

27. Albert A, Müller HR, Hetzel A. Optimized transcranial Doppler technique for the diagnosis of cardiac right-to-left shunts. $J$ Neuroimaging. 1997;7:159-163.

28. Blersch W, Draganski B, Holmer S, Koch H, Schlachetzki $F$, Bogdahn U, et al. Transcranial duplex sonography in the detection of patent foramen ovale. Radiology. 2002;225:693-699.

29. Droste DW, Reisener M, Kemény V, Dittrich R, SchulteAltedorneburg G, Stypmann J, et al. Contrast transcranial Doppler ultrasound in the detection of right-to-left shunts. Reproducibility, comparison of 2 agents, and distribution of microemboli. Stroke. 1999;30:1014-1018.

30. Ferrarini G, Malferrari G, Zucco R, Gaddi O, Norina M, Pini LA, et al. High prevalence of patent foramen ovale in migraine with aura. J Headache Pain. 2005;6:71-76.

31. Heckmann JG, Niedermeier W, Brandt-Pohlmann M, Hilz MJ, Hecht M, Neundörfer B, et al. Detection of patent foramen ovale. Transesophageal echocardiography and transcranial Doppler sonography with ultrasound contrast media are "supplementary, not competing, diagnostic methods". Med Klin. 1999;94:367-370.

32. Horner S, Ni XS, Weins W, Harb S, Augustin M, Duft M, et al. Simultaneous bilateral contrast transcranial Doppler monitoring in patients with intracardiac and intrapulmonary shunts. J Neurol Sci. 1997;150:49-57.

33. Jauss M, Kaps M, Keberle M, Haberbosch W, Dorndorf W. A comparison of transesophageal echocardiography and transcranial Doppler sonography with contrast medium for detection of patent foramen ovale. Stroke. 1994;25:1265-1267.

34. Job FP, Ringelstein EB, Grafen Y, Flachskampf FA, Doherty C, Stockmanns A, et al. Comparison of transcranial contrast Doppler sonography and transesophageal contrast echocardiography for the detection of patent foramen ovale in young stroke patients. Am J Cardiol. 1994;74:381-384.

35. Karnik R, Stöllberger C, Valentin A, Winkler WB, Slany J. Detection of patent foramen ovale by transcranial contrast Doppler ultrasound. Am J Cardiol. 1992;69:560-562.

36. Klötzsch C, Janssen G, Berlit P. Transesophageal echocardiography and contrast-TCD in the detection of a patent foramen ovale: experiences with 111 patients. Neurology. 1994;44:1603-1606.

37. Nemec JJ, Marwick TH, Lorig RJ, Davison MB, Chimowitz MI, Litowitz $\mathrm{H}$, et al. Comparison of transcranial Doppler ultrasound and transesophageal contrast echocardiography in the detection of interatrial right-to-left shunts. Am J Cardiol. 1991;68:14981502.

38. Nygren AT, Jogestrand T. Detection of patent foramen ovale by transcranial Doppler and carotid duplex ultrasonography: a comparison with transoesophageal echocardiography. Clin Physiol. 1998;18:327-330. 
39. Orzan F, Liboni W, Bonzano A, Molinari F, Ribezzo M, Rebaudengo N, et al. Follow-up of residual shunt after patent foramen ovale closure. Acta Neurol Scand. 2010;122:257-261.

40. Sastry S, MacNab A, Daly K, Ray S, McCollum C. Transcranial Doppler detection of venous-to-arterial circulation shunts: criteria for patent foramen ovale. J Clin Ultrasound. 2009;37:276-280.

41. Souteyrand G, Motreff P, Lusson JR, Rodriguez R, Geoffroy E, Dauphin $\mathrm{C}$, et al. Comparison of transthoracic echocardiography using second harmonic imaging, transcranial Doppler and transesophageal echocardiography for the detection of patent foramen ovale in stroke patients. Eur J Echocardiogr. 2006;7:147-154.

42. Venketasubramanian N, Sacco RL, Di Tullio M, Sherman D, Homma S, Mohr JP. Vascular distribution of paradoxical emboli by transcranial Doppler. Neurology. 1993;43:1533-1535.

43. Mojadidi MK, Roberts SC, Winoker JS, Romero J, GoodmanMeza D, Gevorgyan R, et al. Accuracy of transcranial Doppler for the diagnosis of intracardiac right-to-left shunt: a bivariate meta-analysis of prospective studies. JACC Cardiovasc Imaging. 2014;7:236-250.
Conflicto de intereses: Los autores declaran no tener potenciales conflicto de intereses con respecto a la investigación, autoría y/o publicación de este artículo.

Financiamiento: Los autores recibieron apoyo financiero por ARUAL Medicina de Reanimación S.C para la realización y/o publicación de este artículo.

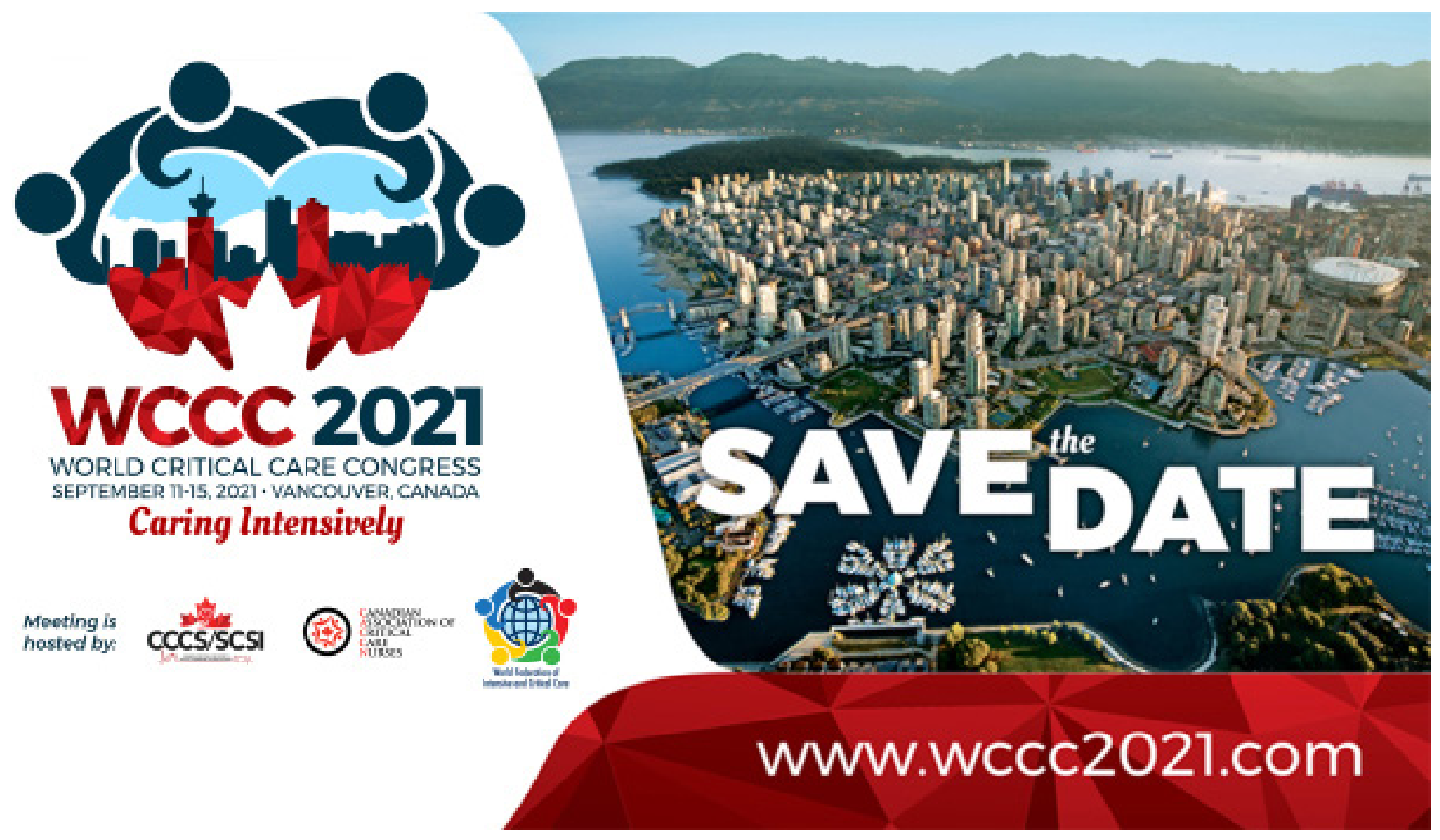

